EFICIENCIA EN LA GESTIÓN FINANCIERA EN EL SUPREMO TRIBUNAL DE JUSTICIA DEL ESTADO DE TAMAULIPAS, MÉXICO ${ }^{1}$

\title{
EFFICIENCY IN THE FINANCIAL MANAGEMENT IN THE SUPREME COURT OF JUSTICE IN THE STATE OF TAMAULIPAS, MÉXICO
}

\author{
David Ortiz González ${ }^{2}$, Mónica Lorena Sánchez ${ }^{3}$ y Yesenia Sánchez Tovar ${ }^{4}$ \\ Universidad Autónoma de Tamaulipas-México
}

RECIBIDO: Agosto 18 de 2014

ACEPTADO: Noviembre 01 de 2014

\section{RESUMEN}

El presente artículo es producto de una investigación, cuyo objetivo consistió en analizar la eficiencia en la gestión financiera en el Supremo Tribunal de Justicia del estado de Tamaulipas, México. De alcance exploratorio, la técnica de investigación utilizada fue revisión literaria y la entrevista, la cual se aplicó a una muestra a conveniencia de este organismo, a través de una guía como instrumento, donde participaron los titulares de las áreas de Recursos Humanos, Finanzas, Almacén y Nóminas. El enfoque de la investigación es cualitativo, el resultado es el análisis de la eficiencia en la gestión financiera en el Supremo Tribunal de Justicia del estado de Tamaulipas, México, en el que se evidenciaron dos factores determinantes (centros de costos e indicadores de seguimiento) que coadyuvan a la integración del cuadro de mando integral, para fortalecer el desempeño de dicho ente público. Se concluye que las finanzas públicas tienen gran importancia, el estudio científico y forma de aplicación técnica componen un factor para la estabilización y crecimiento económico del país, donde la investigación y estructuración en los sistemas de gestión permiten que los entes públicos obtengan los recursos materiales y financieros necesarios para su operación.

Palabras clave: eficiencia, justicia, finanzas, modelo Sistema de Gestión.

\begin{abstract}
This article is a product of an investigation; in which its main objective consists of analyzing the financial management efficiency of the Supreme Court of Justice of the Estado de Tamaulipas, México. An exploratory scope survey was made, using the technique of investigation of literary reviews and interviews, which were applied to a specific sample of this body, through a guide as an instrument, and with the participation of those responsible for Human Resources, Finances, Store and Payment Bureau. The approach of the investigation is qualitative; the result is the analysis of the effectiveness of the financial management of the Supreme Court of Justice of the Estado de Tamaulipas, México. In them, there were demonstrated two main factors (Centers of Costs and Monitoring Indicators) that contributed to the integration of the Balanced Scorecard, to strengthen the performance of the mentioned public entity. We can arrive to a conclusion; actually public finances are of an utmost importance, the scientific study and a form of technical application compose a factor for the stabilization and economic growth of the country, where the investigation and the structure of the management systems allow public entities to obtain material and financial resources that are necessary for their operation.
\end{abstract}

Key words: efficiency, justice, finances, Model of management system.

Este artículo se puede referenciar

Ortiz, D., Sánchez, M. \& Sánchez, Y. (2015). Eficiencia de la gestión financiera en el supremo tribunal de justicia del estado de Tamaulipas, México, en Desarrollo Gerencial Revista de la Facultad de Ciencias Económicas, administrativas y contables de la Universidad Simón Bolívar 7(1), 48-67.

\footnotetext{
${ }^{1}$ Proyecto de Investigación. Sistema Integral Judicial para el fortalecimiento del desempeño y la calidad del servicio prestado por el Supremo Tribunal de Justicia del Estado Tamaulipas" Fue financiado por CONACYT (Consejo Nacional de Ciencia y Tecnología)

${ }^{2}$ Egresado de la Maestría en Dirección Empresarial (PNPC del Conacyt) de la Universidad Autónoma de Tamaulipas, Universidad Autónoma de Tamaulipas, México dao glz@live.com.mx

${ }^{3}$ Doctora en Ciencias de la Administración, profesora investigadora de la Universidad Autónoma de Tamaulipas, miembro del SNI, México msanchel@uat.edu.mx, msanchel@gmail.com

${ }^{4}$ Doctora en Economía y gestión de las organizaciones, profesora investigadora de la Universidad Autónoma de Tamaulipas, miembro del SNI, Méxicoyesanchez@uat.edu.mx
}

Desarrollo Gerencial, 7 (1) pp. 48 - 67 Enero-Junio 2015. ISSN: 2145-5147 (On Line). Universidad Simón Bolívar. 


\section{1. - INTRODUCCIÓN}

Por muchos años las encuestas de opinión han mostrado una imagen negativa y deteriorada de los tribunales a los ojos de la población. Por ejemplo, en Alemania, Portugal, España y Estados Unidos de América las encuestas realizadas en distintos momentos y lugares demuestran que no todo tiempo pasado fue mejor (Fix-Fierro, 2006). De acuerdo con una encuesta realizada en la ciudad de México en 1996, la gran mayoría de los consultados pensaba que los juicios son excesivamente lentos, demasiados costosos y apenas justos; más de una tercera parte de los indagados expresaron un concepto desfavorable (Covarrubias y Aociados, 1996).

No obstante, resulta con graves dificultades para valorar la producción pública, ya que, a diferencia de lo que ocurre en el sector privado, no existe una definición de lo que es un producto público y no existe un precio de venta como tal que considere el producto público en cuanto beneficio social obtenido por el servicio prestado, y precisamente por su condición de bien público, dichos bienes no tienen precio de venta (Stiglitz, 2003). Cabe destacar, que en el sector público parte de la producción es intangible, lo cual dificulta su cuantificación y, además, no se vende en el mercado, razón por la que no existe un precio para valorar y medir la sanidad, educación, el servicio judicial, entre otros (Rueda, 2011).

Al respecto, Cordero (2005) expone que la acelerada evolución que muestran los cambios del mundo moderno, ya sea en el sector público o privado, han generado una corriente a nivel mundial que se dirige hacia la renovación y el progreso de gestión; cambios que deben ser justificados, ineludibles e inaplazables a todos los niveles y con una particularidad: deben darse de manera profunda y acelerada; bajo esta premisa uno de los propósitos fundamentales del progreso de la gestión, es convertir a la Administración Pública en una misión de servicio a la sociedad, en condiciones de transparencia, disciplina y control para alcanzar niveles óptimos de eficiencia, eficacia y efectividad.

Por su parte, Mora y Vivas (2001) destacan que hoy en día resulta muy evidente la necesidad de llevar una gestión más profesional y preocupada por la economía, la eficiencia, la eficacia y la efectividad de las acciones gubernamentales, que da lugar a lo que se conoce como "nueva Administración Pública. [(...) Por ello es] de vital importancia para cualquier sector público conocer el nivel de eficiencia con el que produce los servicios públicos, ya que cuanto más eficiente sea, mayor ahorro de recursos conseguirá y, por tanto, menos gasto será necesario para garantizar una cantidad determinada de servicios públicos a la sociedad" (Rueda, 2011).

Anualmente, se dan a conocer los resultados de la Encuesta Nacional de Victimización y Percepción sobre Seguridad Pública (Enpive), realizada regularmente los meses de marzo y de abril por (Inegi) el Instituto Nacional de Estadística y Geografía (2012). La razón de ser de la Envipe es proporcionar a la 
sociedad información a nivel nacional y por entidades acerca de los problemas delictivos durante los periodos antes mencionados y sobre la percepción social respecto de la seguridad pública y el desempeño de las autoridades, así como proporcionar elementos a los diferentes órdenes de gobierno que sirvan de apoyo en los procesos de política pública.

Los resultados de la encuesta mostraron el nivel de confianza por parte de la sociedad en cuanto al sistema judicial del estado de Tamaulipas, que tuvo inicialmente un $4 .^{\circ}$ lugar a nivel nacional en el 2011, y luego ocupó la posición $17 .^{\circ}$ en 2012. A su vez, la percepción del desempeño de los jueces fue de un $7^{\circ}$ lugar y en el 2012 pasó al puesto $15^{\circ}$.

Por lo anterior, es de gran preocupación que en un año haya habido un retroceso significativo en la percepción de la sociedad, a pesar que el Poder Judicial del estado de Tamaulipas es un organismo que se ha certificado desde el año 2009 conforme a la norma ISO 9001:2008 en los procesos de la impartición de justicia, aunque cabe señalar que se tuvo un cambio en la metodología de la aplicación de esta encuesta lo cual pudo ocasionar estos cambios.

De acuerdo con la Envipe, es indispensable llevar a cabo un plan que reduzca la negativa percepción de la sociedad hacia este organismo, a fin de mejorar la imagen desfavorable que tienen los ciudadanos. Por los resultados ya mencionados, el Supremo Tribunal de Justicia del Estado (STJE) decidió indagar en las causas de este retroceso tan marcado en muy poco tiempo. Por ello, esta investigación contribuirá a identificar el origen de semejante repliegue, haciendo énfasis en:

¿Cómo aplicar la eficiencia en la gestión financiera en el Supremo Tribunal de Justicia estado de Tamaulipas, México?

Esta investigación se justifica porque coadyuvará al cumplimiento de los artículos de la Ley General de Contabilidad (2008), además de que propondrá herramientas como los indicadores, para mejorar la eficiencia de la gestión financiera en el STJET. No obstante, también apoyará al inicio de investigaciones sobre el área de finanzas, que según Hidalgo (2007) y Gómez (2005) señalan el abandono de investigación en el ámbito de las finanzas y la contabilidad de las organizaciones, pues dicha indagación evidenciará las diversas situaciones que podían haberse evadido si se hubiese dispuesto de la suficiente información financiera útil. En caso de muchos cambios, deberán implementarse en dicha área para prevenir las crisis en el futuro y resolver más eficazmente la actual.

Como limitaciones, se menciona que este estudio se concreta en la eficiencia de la gestión financiera del STJET. Se entiende por eficiencia de la gestión financiera la adecuada toma de decisiones orientada a convertir los recursos disponibles en recursos productivos y generadores de valor permitiendo el alcance de los objetivos estratégicos de la empresa (Kaplan \& Norton, 2009). Para dicho propósito, se analiza 


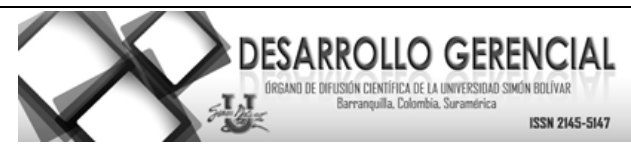

solamente este ente y la percepción de los funcionarios que brindan dichos servicios, por lo que es un limitante el hecho de que no se den generalizar los resultados obtenidos a otras organizaciones.

Del mismo modo, es necesario indicar que la realización de este trabajo siguió la técnica de investigación de la entrevista donde se plantearon preguntas detonadoras que, a su vez, generaron preguntas derivadas; así, el entrevistador pudo modificar o agregar preguntas conforme avanzó la entrevista y se deseó obtener información adicional, de esta forma se siguió lo sugerido por Yin (2009) referente a que la entrevista más que preguntas estructuradas y rígidas debe ser una conversación guiada.

Por su parte, González (2008) señala que el éxito de la gestión de las entidades no lucrativas debe medirse por la eficacia y eficiencia de su gestión en la satisfacción de las necesidades de sus usuarios y no por los resultados financieros obtenidos.

Aunado a lo anterior, se debe considerar que la eficiencia de una entidad gubernamental no debe medirse por la maximización de sus ingresos o la minimización de sus gastos, sino por la calidad e intensidad con que realice los propósitos públicos (Placencia, 2012), la presente investigación ayudará a determinar bajo qué indicadores puede medirse la eficiencia en la gestión financiera del STJET. Estos indicadores son el resultado de la investigación y serán explicados en el cuadro 7.

\section{2.-MÉTODO}

\section{Diseño}

Para este estudio se utilizó un tipo de diseño de investigación transversal, lo cual implica que la entrevista fue aplicada en un momento único y no a través del tiempo (Hernández, 1997), y de alcance exploratorio; los datos obtenidos se examinaron mediante el método de análisis de contenido de acuerdo a Hernández, Fernández y Baptista (2007), y se documentó la información más sobresaliente organizándola en categorías. Hay un procedimiento para otorgar y ejercer el presupuesto, -las partidas que ejecuta el presupuesto, los centros de costos del STJET del que se derivan los mismos por capítulo-, así como está lo relacionado con el presupuesto autogenerado y el Fondo Auxiliar. Este estudio no establece una relación-causa; por lo tanto, no se especifican las variables.

Las fuentes de información fueron los titulares de las áreas de Finanzas, Nóminas, Recursos Humanos y Almacén de la ciudad de Victoria, Tamaulipas (México), sujetos en su ambiente natural (de trabajo) sin manipular o provocar sus comportamientos para posteriormente proceder a analizarlos (Kerlinger, 1975). 


\section{Participantes}

Los actores de este estudio fueron los titulares de las áreas de Finanzas, Nóminas, Recursos Humanos y Almacén, cuyo perfil se describe en la tabla 1; se les identifica como personas clave en la medida en que poseen la información requerida para este trabajo, en especial los cuatro sujetos que la proporcionaron y que fueron seleccionados considerando que era precisamente la que se necesitaba, pues el análisis de la gestión financiera involucra en cuestión las actividades desarrolladas en dichas áreas.

Tabla 1. Perfil de los entrevistados

\begin{tabular}{|l|c|c|c|c|}
\hline \multicolumn{1}{|c|}{$\begin{array}{c}\text { Puesto } \\
\text { Directores de: }\end{array}$} & Nivel educativo & $\begin{array}{c}\text { Antigüedad en } \\
\text { el puesto }\end{array}$ & $\begin{array}{c}\text { Antigüedad en } \\
\text { la institución }\end{array}$ & Edad \\
\hline Finanzas & Maestría & 14 & 16 & 53 \\
\hline Nóminas & Licenciatura & 7 & 7 & 35 \\
\hline Recursos Humanos & Maestría & 16 & 21 & 54 \\
\hline Almacén & Maestría & 11 & 19 & 42 \\
\hline
\end{tabular}

Nota: elaboración propia.

\section{Instrumentos}

Se aplicaron dos técnicas de investigación: la revisión de literatura (leyes, artículos e investigaciones científicas) y la entrevista semiestructurada a cada uno de los elementos de la muestra, que se seleccionó por conveniencia, y atendiendo las consideraciones de su condición clave de acuerdo a Munch (2007). El instrumento aplicado fue una guía de entrevista donde se plantearon cuatro preguntas ejes o detonadoras que, a su vez, generaron preguntas derivadas; así, el entrevistador pudo modificar o agregar preguntas conforme avanzó la entrevista y se deseó obtener información adicional, de esta forma se siguió lo sugerido por Yin (2009) referente a que la entrevista más que preguntas estructuradas y rígidas debe ser una conversación guiada. La técnica de investigación fue la entrevista semiestructurada, que se utilizó a fin de obtener información con la que se pudiera acercar mejor al problema observado. Para ello se elaboró una guía (anexo 1).

A partir de las entrevistas aplicadas en la evaluación de la gestión financiera, se realizó una matriz de evidencia (anexo 2), para analizar las respuestas de las cuatro personas clave indagadas, buscando así determinar observaciones generales que aunadas a la información documental prestada por la institución permitiera generar indicadores de eficiencia en la gestión financiera.

\section{Procedimientos}

El trabajo de campo se desarrolló en el marco de la revisión de la literatura y documentos inherentes a la unidad de estudio, como el Programa Estratégico de Desarrollo Institucional del STJET y la Ley 


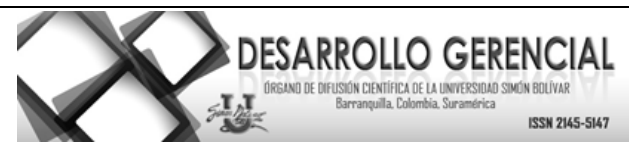

Orgánica del Poder Judicial del estado de Tamaulipas (2013). Así mismo para la recolección de información en el campo, se aplicaron entrevistas, pero con la libertad de introducir ciertas preguntas que se consideraron pertinentes, en especial la realizada para el proceso de investigación a los jefes de área (4), en el periodo de agosto-diciembre 2013.

Este estudio se adelantó en cuatro fases: 1. Antes de aplicar cada entrevista se hizo la presentación de los investigadores al entrevistado, para explicarle el objetivo buscado y el carácter académico que revestía. 2. A quienes lo requirieron, se les solicitó mediante oficio conceder las declaraciones. 3. Las entrevistas fueron grabadas con autorización de los informantes. 4. Por último, se dio el análisis del contenido entregado.

\section{3.- RESULTADOS}

El tipo de resultado es analítico (Yin, 2009), para mostrar la eficiencia en la gestión financiera en el STJET destacándose:

Del procedimiento para el otorgamiento y ejercicio del presupuesto, se tiene que solo utiliza el presupuesto en nóminas y materiales para la toma de decisiones, no obstante, es posible calcular los costos por actividades o por áreas, los cuales no son determinados ni utilizados para la toma de decisiones; otro aspecto es lo referente a indicadores tanto de seguimiento como los obligados por Ley General de Contabilidad Gubernamental (2013), sin embargo, en la actualidad no son calculados por el STJET. Esta información se utilizó para la eficiencia en la gestión financiera en el STJET (México), para lo cual se evidenciaron los factores de la perspectiva financiera

De las partidas que ejecuta el presupuesto, de acuerdo a las áreas en el STJET, se consideran como centros de costos las áreas de Presidencia, Consejo de Adjudicatura, el Tribunal Electoral, Salas, Juzgados, Áreas Administrativas y Proyectos. En el siguiente cuadro (1) puede observarse el nivel de presupuesto dado, lo que representa el nivel de costos, medidos en porcentajes de acuerdo al presupuesto dado del año 2012.

Cuadro 1. Presupuesto de las áreas del STJET del año 2012

\begin{tabular}{l|l|l}
\hline Área & Porcentaje 2012 (\%) & Presupuesto 2013 (\%) \\
\hline Presidencia & 13.12 & 14.06 \\
\hline Consejo de Adjudicatura & 2.75 & 2.85 \\
\hline Tribunal Electoral & 4.52 & 4.88 \\
\hline Salas & 16.01 & 15.43 \\
\hline Juzgados & 42.68 & 46.83 \\
\hline
\end{tabular}




\begin{tabular}{l|l|l}
\hline Áreas Administrativas & 16.06 & 15.11 \\
\hline Proyectos & 4.86 & 0.85 \\
\hline
\end{tabular}

Nota: elaboración propia.

Del presupuesto destinado a cada área, se desglosa su respectiva asignación del siguiente cuadro (2).

Cuadro 2. Presupuesto por capítulo del STJET

\begin{tabular}{l|l|l}
\hline Capítulo & $\mathbf{2 0 1 2}(\mathbf{\%})$ & $\mathbf{2 0 1 3}(\mathbf{\%})$ \\
\hline Servicios Personales & 79.69 & 84.41 \\
\hline Materiales y Suministros & 3.94 & 3.44 \\
\hline Servicios Generales & 11.75 & 9.43 \\
\hline Transferencias subsidios, asignaciones & 0.29 & 0.32 \\
\hline Bienes muebles, inmuebles e intangibles & 2.29 & 2.28 \\
\hline Inversión Pública & 2.04 & 0.12 \\
\hline
\end{tabular}

Nota: elaboración propia.

Con respecto a los centros de costos, las Salas y Juzgados representan los centros de costos más importantes en el STJET, ya que entre ambos representan alrededor del $60 \%$ del presupuesto ejercido en ambos años, porque además estos son los encargados del cumplimento de la misión del Supremo: la impartición de justicia. Para un análisis más detallado de estas dos áreas se observa a continuación la información obtenida del presupuesto por capítulo de uno y otro en los siguientes cuadros ( 3 y 4 ).

Cuadro 3. Presupuesto por capítulo de Juzgados del STJET

\begin{tabular}{l|l|l}
\hline Capítulo & $2012(\%)$ & $2013(\%)$ \\
\hline Servicios Personales & 87.23 & 87.35 \\
\hline Materiales y Suministros & 3.11 & 2.80 \\
\hline Servicios Generales & 9.05 & 8.60 \\
\hline Transferencias subsidios, asignaciones & 0 & 0 \\
\hline Bienes muebles, inmuebles e intangibles & 0.61 & 1.24 \\
\hline Inversión Pública & 0 & 0 \\
\hline
\end{tabular}

Nota: elaboración propia.

Cuadro 4. Presupuesto por capítulo de Salas del STJET

\begin{tabular}{l|l|l}
\hline Capítulo & $2012(\%)$ & $2013(\%)$ \\
\hline Servicios Personales & 95.93 & 94.62 \\
\hline Materiales y Suministros & 0.17 & 1.47 \\
\hline Servicios Generales & 3.65 & 3.37 \\
\hline Transferencias, Subsidios, Asignaciones & 0 & 0.00 \\
\hline
\end{tabular}




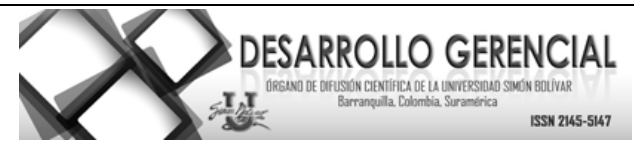

\begin{tabular}{l|l|l} 
Bienes Muebles, Inmuebles e Intangibles & 0.25 & 0.54 \\
\hline Inversión Pública & 0 & 0.00 \\
\hline
\end{tabular}

Nota: elaboración propia.

Del presupuesto autogenerado por el STJET, se determinó que esta fuente de recursos tiene en el STJET dos formas de ingreso: por medio del Fondo Auxiliar y por la participación en programas del Gobierno Federal. El STJET utiliza el Fondo Auxiliar para la Administración de Justicia para mejora de las condiciones de trabajo de los servidores públicos del Poder Judicial, mediante la aplicación de estímulos y recompensas, y su capacitación; adquisición de mobiliario y equipo, libros y demás material de contenido jurídico siempre que aumente el cumplimiento eficaz de las funciones. El Fondo Auxiliar recaudado en años anteriores se refleja en el cuadro (5).

Cuadro 5. Fondo Auxiliar, recaudo 2011-2013

\begin{tabular}{c|c}
\hline Año & Fondo \$ (en MXP) \\
\hline 2013 & $20,556,869.51$ \\
\hline 2012 & $18,548,631.57$ \\
\hline 2011 & $18,235,762.89$ \\
\hline
\end{tabular}

Nota: elaboración propia.

El Fondo Auxiliar para la Administración de la Justicia es recaudado principalmente de acuerdo con la Ley Orgánica del Poder Judicial del estado de Tamaulipas (2013) por: I. Fondos propios constituidos, II. Fondos ajenos constituidos por depósitos en efectivo o en valores, que por cualquier causa se realicen o se hayan realizado ante los tribunales judiciales del fuero común del Estado.

Una peculiaridad importante en esta partida es que solamente ocho estados de la república mexicana (Sinaloa, Yucatán, México, Veracruz, Guerrero, Guanajuato, Michoacán y Tamaulipas) cuentan con autonomía tanto para distribuir y ejercer su presupuesto de esta forma como para generar un recurso propio. El artículo 107 de la Constitución Política de Tamaulipas señala que el Poder Judicial cuenta con autonomía presupuestal, orgánica y funcional. El presupuesto es el suficiente para cubrir las necesidades en el ejercicio de su función. Además, el Poder Judicial constituye un Fondo Auxiliar para la Administración de la Justicia en los términos previstos en la ley.

Donde el Poder Ejecutivo del Estado cuida que las cantidades que integran este Fondo se entreguen trimestralmente al Poder Judicial, que a través de su presidente tendrá la obligación de dar a conocer lo recaudado en el año anterior cuando rinda el informe anual sobre el estado que guarda la administración de justicia, y dentro de los informes trimestrales de cuenta pública correspondientes, los que remiten dentro de la segunda quincena del mes siguiente a la terminación de cada trimestre, para su revisión por el 


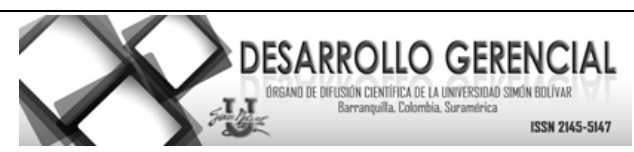

Congreso del Estado. La administración de la justicia del STJET se engloba dentro de la Administración Pública.

\section{4.- DISCUSIÓN}

Como se observa en la matriz de evidencia de la entrevista (anexo 2), de las cuatro áreas entrevistadas el presupuesto se realiza con base histórica, en donde el $80 \%$ va dirigido a la atención de Juzgados y Salas, asignación según la cual deja entrever que el objetivo de la organización es impartir justicia; sin embargo, no está relacionado con metas específicas. Las áreas financieras no trabajan con indicadores de eficiencia, solo llevan controles internos que necesariamente hay que alinear a objetivos institucionales.

El conocimiento de la gestión financiera del Poder Judicial permite concluir que existe un problema en la organización en la medida en que aún no se adecúa a las teorías de la nueva Administración Pública. La principal característica de este último modelo es su énfasis en las normas y valores económicos (Christensen \& Lagreid, 2001).

Arellano y Cabrero (2005) señalan que la nueva gestión pública (NGP) propone un Gobierno fuerte y formal siempre, y que actúe racionalmente. La insatisfacción de los ciudadanos ante la incapacidad de prestación de servicios de la Administración, así como los esfuerzos de los socios y de los inversionistas internacionales obligan al ejecutivo a orientar el tema del "buen gobierno" en una dirección que garantice más y mejor servicio, evite el mal uso de recursos y ponga un fin a su derroche. Con una NGP que funcione adecuadamente, los desarrollos erráticos como la corrupción y el nepotismo se detectarán con facilidad y de manera oportuna de tal manera que podrán ser evitados (Schroder, s.f.).

El Supremo Tribunal de Justicia en Tamaulipas necesita que su gestión financiera responda a los objetivos estratégicos planteados por la organización. En la nueva gerencia pública, se considera el uso de herramientas de gestión estratégica, como el cuadro de mando integral (CMI), que aun cuando nacen para uso de la iniciativa privada pueden ser aplicadas en los entes de carácter público (Kaplan \& Norton, 1992). Por esta razón, el análisis de la situación del Poder Judicial en Tamaulipas obliga a la entidad a la aplicación de una herramienta como esa.

En este sentido, el CMI puede ser el sistema idóneo que permita examinar los resultados obtenidos, por cuanto mantiene no solo las medidas financieras, sino también las complementa con la evaluación de otras perspectivas: la del cliente, la de procesos internos y la de aprendizaje y crecimiento donde, por ejemplo, utiliza un nuevo lenguaje que describe los elementos clave para el cumplimiento de la estrategia (Niven, 2003; Kaplan \& Norton, 2009). 
Cuadro 6. Dimensiones del cuadro de mando integral

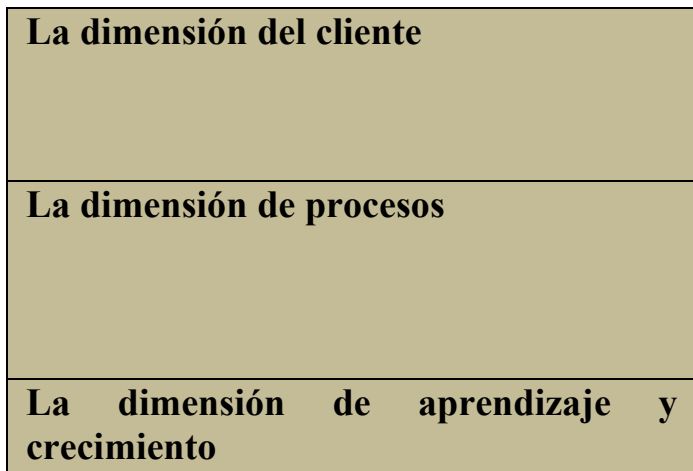

La dimensión financiera
En esta dimensión se recogen los objetivos establecidos en relación con los clientes de la organización conforme sus necesidades, para así llegar a los objetivos financieros.

Identifica aquellos procesos que se espera tengan un fuerte impacto en la consecución de la estrategia, que conjuntamente con los de aprendizaje y crecimiento facilitarán la consecución de los objetivos de procesos.

Esta dimensión identifica los activos intangibles críticos para la consecución de la estrategia y deben estar agrupados y alineados con los objetivos de proceso.

Recoge las necesidades de satisfacer en forma económica las metas institucionales mientras enfoca los objetivos e indicadores del resto de las dimensiones.

Nota: elaborado a partir de Amo (2010).

El uso de un instrumento de perfil gerencial como el CMI sirve como medio de ejecución de un práctico control de la diligencia estatal, que permite concertar los objetivos de perfeccionamiento (dimensión de aprendizaje y crecimiento) y racionalización de los servicios públicos (dimensión de los procesos internos), a efecto de que la utilización de los recursos sea con costos mínimos y, consiguientemente, con minúsculos déficits (dimensión financiera) dentro de una perspectiva orientada a los resultados; en otras palabras, procurar la maximización de la satisfacción de los ciudadanos (perspectiva de los clientes). Asimismo, por sus características de instrumento de control estratégico, entiende ser apropiado para monitorear la ejecución de los planes del Gobierno y su comunicación a todo el aparato administrativo gubernamental mediante el surgimiento de nuevos CMI en los niveles inferiores de la Administración Pública alineados con el CMI original (Barros \& Rodríguez, 2004).

La dimensión financiera en los organismos públicos desarrolla aspectos distintos ya que, por un lado, no constituye precisamente un objetivo (Barros \& Rodríguez, 2004), sino que reviste un carácter restrictivo en función de la disponibilidad de recursos. Desde otro punto de vista se orienta al mantenimiento del equilibrio presupuestario; además, permite plantear objetivos de reducción de costos y de eficiencia en la recaudación (Kaplan \& Norton, 1997).

Realmente los éxitos en las organizaciones públicas como es el caso del Supremo Tribunal de Justicia del estado de Tamaulipas deben medirse en razón de cómo atienden a las necesidades de los ciudadanos. Así, dentro del Gobierno esta perspectiva enfatiza la eficiencia en costos, es decir, la habilidad para ofrecer máximo valor a la comunidad con el mínimo gasto (Holguín, 2010), en otras palabras: alcanzar la meta de la manera más eficiente. Para tal efecto, es relevante comprender que el costo en el sector público 


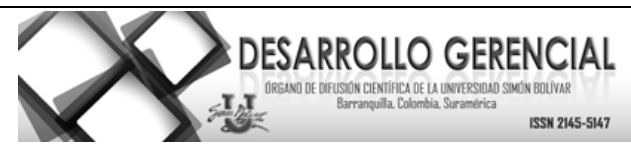

se refiere a los insumos con los que necesariamente hay que dotar a las instituciones públicas para que estén en capacidad de prestar bienes y servicios (Calle \& Makón, 2012).

Se pudo concluir que el diseño o la selección de indicadores en la elaboración de CMI es una de las etapas más importantes para mejorar la gestión financiera dentro de la organización; el indicador diseñado o establecido debe ser capaz de medir las estrategias definidas, que en el caso del STJET requiere de la implementación de ciertos indicadores para el respectivo logro. El objetivo general que se planteó en esta investigación fue determinar los indicadores que servirán para una mejora en la gestión financiera en el Supremo Tribunal de Justicia; a partir de este se derivó el que busca identificar los factores determinantes para la perspectiva financiera en el STJET. Fue a través de esos objetivos que se realizó este estudio.

Estos objetivos llevaron a que los factores determinantes de la perspectiva financiera para el STJET fuera primeramente la señalización de los centros de costos principales en esta organización, de los cuales resultaron altamente significativas las áreas de Juzgados y Salas, pues su presupuesto ronda el 60\% del total aprobado a todo el ente público. Es de relevancia para el STJET que el presupuesto global designado y principalmente a estas dos áreas sea manejado de una manera eficiente y eficaz; esto se puede lograr mediante el seguimiento continuo del manejo del recurso dado para el control adecuado sin que exista un sobregasto inesperado o anormal en este ente público.

Aunque el STJET tiene por ley un presupuesto que le otorga el estado de Tamaulipas, esta dependencia también cuenta con la autogeneración de recursos económicos, ya sea por medio del Fondo Auxiliar mediante diversas situaciones o participando con proyectos en programas o convocatorias del Gobierno Federal; la medición de este recurso en comparación con el presupuesto general dado resulta importante ya que forma un indicador que apoyará la optimización de la gestión financiera en esta organización. Con base en esto se proponen indicadores para la mejora de la gestión financiera respondiendo así a la pregunta de investigación.

Del análisis de estos indicadores se destaca la visualización de la mejora en la gestión financiera del STJET, ya que su grado de eficiencia y eficacia tanto en el presupuesto ejercido como en comparación con la eficiencia de los casos resueltos ha aumentado de un año a otro (2012-2013) de manera significativa, notándose así que pueden establecerse nuevas metas.

\section{Propuesta de indicadores para la eficiencia en la perspectiva financiera STJET}

En México, en general existen pocos controles para garantizar una adecuada gestión judicial que permita cumplir el objetivo por el cual fueron constituidos: servir, mantener el orden y resolver adecuadamente los conflictos que se generen entre los individuos que conforman una sociedad, 
permitiendo el acceso a la impartición de justicia a toda la ciudadanía, cuidando reducir los costos y los tiempos de demora en todos los procesos que contempla la Constitución para la ejecución de los juicios, y dictando al mismo tiempo resoluciones en forma equitativa y eficiente.

Por ello, hay un dilema en el funcionamiento de las instituciones judiciales, pues a la vez que deben alcanzar el objetivo, dado el creciente nivel de demanda de los servicios requeridos por la sociedad, presentan insuficiente capacidad y recursos para lograrlo.

Como respuesta a este dilema, la presente investigación plantea el establecimiento de nuevos sistemas de desempeño y evaluación.

Dicho lo anterior es necesario focalizar la atención en la administración adecuada del servicio de impartición de justicia con el fin de lograr una utilización más eficiente de los recursos asignados. Los resultados de esta investigación permiten proponer un modelo de evaluación financiera. El modelo incorpora indicadores y mecanismos de planeación, organización y control que pueden funcionar como sistemas complejos autoadaptables, con la finalidad de mejorar el funcionamiento de la administración de las instituciones judiciales. A continuación se proponen una serie de indicadores para mejorar la eficiencia y eficacia del STJET, en cuanto a la perspectiva financiera.

Cuadro 7. Indicadores de medición de la perspectiva financiera del STJET

\begin{tabular}{|c|c|c|c|c|c|c|c|c|}
\hline $\begin{array}{c}\text { Objetivo } \\
\text { estratégico }\end{array}$ & $\begin{array}{l}\text { Nombre del } \\
\text { indicadores }\end{array}$ & Categoría & $\begin{array}{c}\text { Fórmula del } \\
\text { indicador }\end{array}$ & $\begin{array}{c}\text { Unidad } \\
\text { de } \\
\text { medida }\end{array}$ & Frecuencia & Meta & $\begin{array}{l}\text { Acciones } \\
\text { tácticas }\end{array}$ & Responsable \\
\hline \multirow[t]{2}{*}{$\begin{array}{l}\text { Eficiencia del } \\
\text { presupuesto }\end{array}$} & $\begin{array}{l}\text { Presupuesto } \\
\text { total } \\
\text { ejercido }\end{array}$ & 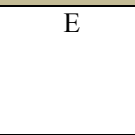 & $\begin{array}{l}\text { (Presupuesto } \\
\text { ejercido/presupuesto } \\
\text { autorizado) *100 }\end{array}$ & $\%$ & Anual & $98 \%$ & 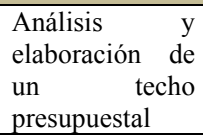 & $\begin{array}{l}\text { Dirección de } \\
\text { Finanzas }\end{array}$ \\
\hline & $\begin{array}{l}\text { Eficiencia } \\
\text { del } \\
\text { presupuesto } \\
\text { ejercido }\end{array}$ & $E$ & $\begin{array}{l}((\% \text { del presupuesto } \\
\text { total ejercido }) / \text { (total } \\
\text { de casos/total de } \\
\text { casos } \\
\text { concluidos }) * 100\end{array}$ & $\%$ & Anual & $110 \%$ & $\begin{array}{l}\text { Análisis } r \\
\text { elaboración de } \\
\text { un techo } \\
\text { presupuestal }\end{array}$ & $\begin{array}{l}\text { Dirección de } \\
\text { Finanzas }\end{array}$ \\
\hline \multirow[t]{3}{*}{$\begin{array}{l}\text { Autogeneración } \\
\text { de recursos }\end{array}$} & $\begin{array}{l}\text { Recursos } \\
\text { generados } \\
\text { por Fondo } \\
\text { Auxiliar }\end{array}$ & $\mathrm{E}$ & $\begin{array}{l}\text { Total del Fondo } \\
\text { Auxiliar } \\
\text { presupuesto ejercido) }\end{array}$ & $\%$ & Anual & $4.8 \%$ & $\begin{array}{l}\text { Sistematización } \\
\text { con ayuda de } \\
\text { apps para el } \\
\text { Fondo Auxiliar }\end{array}$ & $\begin{array}{l}\text { Dirección del } \\
\text { Fondo Auxiliar } \\
\text { para la } \\
\text { Administración } \\
\text { de la Justicia }\end{array}$ \\
\hline & \multirow[t]{2}{*}{$\begin{array}{l}\text { Recursos } \\
\text { para } \\
\text { proyectos } \\
\text { por } \\
\text { Gobierno } \\
\text { Federal }\end{array}$} & $E$ & $\begin{array}{l}\text { (Proyectos federales } \\
\text { metidos } \\
\text { convocatorias/ } \\
\text { proyectos ganadores) } \\
* 100\end{array}$ & $\%$ & Anual & - & $\begin{array}{l}\text { Elaboración de } \\
\text { proyectos con } \\
\text { participaciones } \\
\text { académicas y } \\
\text { profesionales en } \\
\text { su elaboración a } \\
\text { través de } \\
\text { convenios }\end{array}$ & $\begin{array}{l}\text { Planeación y } \\
\text { Desarrollo } \\
\text { Administrativo }\end{array}$ \\
\hline & & $\mathrm{E}$ & $\begin{array}{lrr}\text { Recurso dado } & \text { para } \\
\text { proyectos por } & \text { Gob. } \\
\text { Federal/total } & \text { de } \\
\text { presupuesto } & \\
\text { autorizado) } * 100 & \end{array}$ & $\%$ & Anual & - & $\begin{array}{l}\text { Elaboración de } \\
\text { proyectos con } \\
\text { participaciones } \\
\text { académicas y } \\
\text { profesionales en } \\
\text { su elaboración a } \\
\text { través de } \\
\text { convenios }\end{array}$ & $\begin{array}{l}\text { Planeación y } \\
\text { Desarrollo } \\
\text { Administrativo }\end{array}$ \\
\hline
\end{tabular}




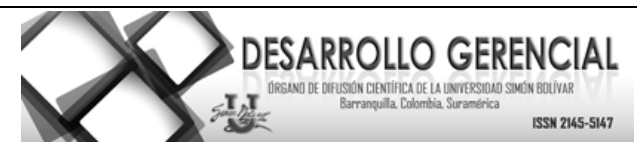

\begin{tabular}{|c|c|c|c|c|c|c|c|c|}
\hline \multirow[t]{3}{*}{$\begin{array}{l}\text { Minimizar los } \\
\text { costos medios } \\
\text { por causa } \\
\text { concluida }\end{array}$} & $\begin{array}{l}\text { Costo } \\
\text { medio por } \\
\text { asunto } \\
\text { resuelto en } \\
\text { salas }\end{array}$ & E & $\begin{array}{ll}\text { (Presupuesto } & \\
\text { ejercido/total de } \\
\text { causas concluidas en } \\
\text { salas) }\end{array}$ & $\$$ & Anual & $\$ 1,000$ & $\begin{array}{l}\text { Revisión y } \\
\text { control } \\
\text { periódico de los } \\
\text { indicadores de } \\
\text { control interno. }\end{array}$ & $\begin{array}{l}\text { Dirección de } \\
\text { Finanzas }\end{array}$ \\
\hline & $\begin{array}{l}\text { Costo } \\
\text { medio por } \\
\text { asunto } \\
\text { concluido } \\
\text { en juzgados }\end{array}$ & $\mathrm{E}$ & $\begin{array}{l}\text { (Presupuesto } \\
\text { ejercido/total de } \\
\text { causas concluidas en } \\
\text { juzgados) }\end{array}$ & $\$$ & Anual & $\$ 3,000$ & $\begin{array}{l}\text { Revisión y } \\
\text { control } \\
\text { periódico de los } \\
\text { indicadores de } \\
\text { control interno. }\end{array}$ & $\begin{array}{l}\text { Dirección de } \\
\text { Finanzas }\end{array}$ \\
\hline & $\begin{array}{l}\text { Costo } \\
\text { medio por } \\
\text { asunto } \\
\text { resuelto }\end{array}$ & $\mathrm{E}$ & $\begin{array}{l}\text { (Presupuesto } \\
\text { ejercido/total de } \\
\text { causas concluidas en } \\
\text { juzgados) }\end{array}$ & $\mathrm{S}$ & Anual & $\$ 6,000$ & $\begin{array}{l}\text { Revisión y } \\
\text { control } \\
\text { periódico de los } \\
\text { indicadores de } \\
\text { control interno. }\end{array}$ & $\begin{array}{l}\text { Dirección de } \\
\text { Finanzas }\end{array}$ \\
\hline
\end{tabular}

$\mathrm{E}=$ estratégico. $\%=$ porcentual. $\$=$ monetario

Nota: elaboración propia.

\section{Indicadores propuestos para control interno en el Área de Finanzas, Juzgados y Salas}

Se propone asimismo un formato de tabla para control interno de finanzas en cuanto al uso y costo por caso por área y capítulo, además de las áreas de Salas y Juzgados, para apoyar el cumplimiento de los indicadores establecidos desde la perspectiva financiera del CMI.

\section{Cuadro 8. Presupuesto por área administrativa}

\begin{tabular}{l|c|c}
\hline Área & Año & $\begin{array}{l}\% \text { del total del } \\
\text { presupuesto }\end{array}$ \\
\hline Presidencia & $\$$ & $\%$ \\
\hline Consejo de la Judicatura & $\$$ & $\%$ \\
\hline Tribunal & $\$$ & $\%$ \\
Electoral & $\$$ & $\%$ \\
\hline Salas & $\$$ & $\%$ \\
\hline Juzgados Total & $\$$ & $\%$ \\
\hline Áreas Administrativas & $\$$ & $\%$ \\
\hline Proyectos & $\$$ & \\
\hline
\end{tabular}

Nota: elaboración propia.

Hoy en día los organismos públicos al igual que las empresas privadas deben buscar la eficiencia en las operaciones, razón por la cual los recursos destinados a la operación tienen que fomentar la productividad y en el caso del sector público, los beneficios sociales que recibe la población. Específicamente en una institución de justicia como la estudiada en este trabajo, se puede observar la existencia de un sistema que cada día progresa en su operación, pero que para alcanzar la optimización requiere el uso de indicadores que, en el caso de organismos públicos, difícilmente alcanzan la optimización de los recursos, puesto que no reflejan una utilidad financiera como el sector privado. Por lo 


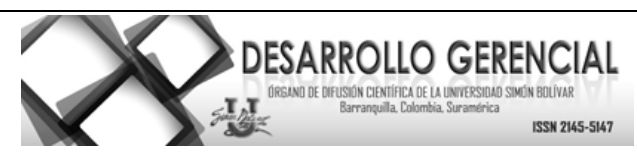

tanto, la única forma de garantizar que una institución pública alcance su resultado (el beneficio social) es a través de su evaluación por medio de indicadores.

Este documento permite identificar diferentes indicadores que facilitan alcanzar los objetivos estratégicos organizacionales.

\section{5.- REFERENCIAS}

Amo, F. (2010). El cuadro de mando integral “Balanced Scorecard”. España: Editorial ESIC.

Arellano, D. \& Cabrero, E. (2005). La nueva gestión pública y su teoría de la organización: ¿Son argumentos antiliberales? Justicia y equidad en el debate organizacional público. Revista Gestión y Política Pública, XIV (3), 599-618.

Barros, J. \& Rodríguez, R. (2004). Una nueva visión del cuadro de mando integral para el sector público. Revista Iberoamericana de Contabilidad de Gestión, 4, 117-148.

Calle, R. \& Makón, M. (2012). La estimación de costos en las entidades del sector público: una propuesta metodológica. Revista Internacional de Presupuesto Público - ASIP, 78 (40), 11- 48.

Covarrubias y Asociados (1996). Entre abogados te veas. Voz y Voto, 41, 23-27.

Christensen, T. \& Lagreid, P. (2001). La nueva Administración Pública: el equilibrio entre la gobernanza política y la autonomía administrativa. Revista Gestión y Política Pública, 10 (1), 55-100.

Fix-Fierro, H. (2006). Tribunales, justicia y eficiencia: Estudio sociojurídico sobre la racionalidad económica en la función judicial. México: Universidad Autónoma de México, Instituto de Investigaciones Jurídicas.

Gómez, F. (2005). Una nota crítica sobre la actual investigación en finanzas. Cuadernos de Economía y Dirección de la Empresa, 24, 105-120.

González, Q. (2008), Los indicadores de gestión y el cuadro de mando en las entidades no lucrativas. Revista de Economía Pública, Social y Cooperativa, 63, 227-252.

Hernández, R. (1997). Metodología de la Investigación. México: Ed. McGraw-Hill.

Hernández, R., Fernández, C. \& Baptista, P. (2007). Metodología de la Investigación (5. a ed.). México: McGraw-Hill.

Hidalgo, E. (2007). Investigación en finanzas y contabilidad: una propuesta de aprendizaje. Libros de Economía y Empresa. España: Deusto. 


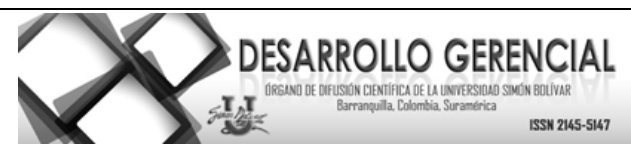

Holguín, B. (2010). Aplicación de un cuadro de mando integral en un organismo del sector público: Caso Banco Central del Ecuador. Tesis de grado no publicada, Escuela Superior Politécnica del Litoral Instituto de Ciencias Matemáticas, Auditoría y Control de Gestión, Ecuador.

Ibarra, M. (2009). Introducción a las finanzas públicas. Cartagena (Colombia): Fundación Universitaria Tecnológica de Comfenalco.

Instituto Nacional de Estadística y Geografía Inegi. (2012). Encuesta Nacional de Victimización y Percepción sobre Seguridad Pública 2012 Envipe: Marco Teórico, Recuperado de: http://www.culturadelalegalidad.org.mx/recursos/Contenidos/Estudiosacadmicosyestadsticos/docu mentos/ENVIPE12\%20Marco\%20Conceptual.pdf.

Kaplan, R. \& Norton, D. (1992). The Balanced Scorecard Measures that drive performance. Boston: Harvard Business School Press.

Kaplan, R. \& Norton, D. (1997). Cuadro de mando integral: The Balanced Scorecard. Barcelona, Gestión 2000.

Kaplan, R. \& Norton, D. (2009). The Balanced Scorecard. Translating strategy into action (3. ${ }^{\mathrm{a}}$ ed.). Boston: Harvard Business School Press.

Kerlinger, F.N. (1975). Investigación del comportamiento: técnicas y metodología. México, D. F.: Nueva Editorial Interamericana.

Mora, A. J. \& Vivas, C. (2001). Nuevas herramientas de gestión pública: el cuadro de mando integral. Madrid: ECA.

Munch, G. (2007). Métodos y técnicas de investigación: Desarrollo de competencias (3. ${ }^{\mathrm{a}}$ ed.). México D.F.: Trillas.

Niven, P. (2003). El cuadro de mando integral paso a paso. Maximizar los resultados Barcelona: Ediciones Gestión 2000.

Placencia, L.A. (2014). Administración General y Administración Pública. Revista Praxis de la Justicia Fiscal y Administrativa, II (5), 128-145.

Rueda, N. (2011). La eficiencia y su importancia en el sector público. Revista Electrónica eXtoikos, 1, 3847.

Ley General de Contabilidad Gubernamental, DOF 09-12/2013, § (2008).

Ley Orgánica del Poder Judicial del Estado, POE Extraordinario 3 07-06/2013, § (2013). 


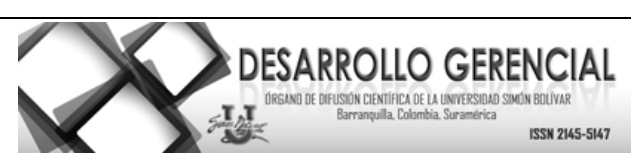

Schröder, P. (S.F.). Nueva gestión pública: Aportes para el buen gobierno. Mexico D.F.: Fundación Friedrich Naumann. Recuperado desde: http://www.freiheit.org/files/534/Schroeder_NGP_Texto_esp.pdf.

Stiglitz, J. E. (2003). La economía del sector público. Barcelona: Editorial Antoni Bosch.

Yin, R. (2009). Case study Research: Design and methods (5. ${ }^{\mathrm{a}}$ ed.). California: SAGE Publications. 


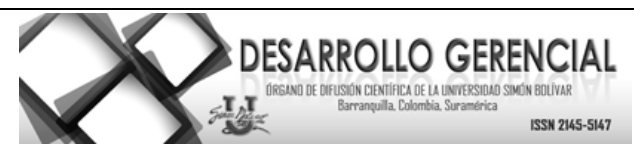

ANEXOS

Anexo 1. Guía de entrevista para los titulares de las áreas de Finanzas, Nóminas, Recursos Humanos y Almacén

\section{Área de Finanzas}

1. ¿El presupuesto ejercido para el Supremo Tribunal de Justicia del estado de Tamaulipas lo tiene dividido por área?

2. ¿Cuáles departamentos ejercen la mayor parte del presupuesto asignado al Tribunal?

3. ¿Qué porcentaje representa cada uno del presupuesto?

4. ¿Cuántos empleados están actualmente en nómina?

5. ¿Qué cantidad del presupuesto es la que está asignada a Almacén?

6. ¿Cuál fue el presupuesto asignado al área de Recursos Humanos?

7. ¿Cuenta con los cálculos de los costos más representativos de cada departamento?

8. ¿Cuáles son?

9. ¿Maneja indicadores de gestión financiera?

10. ¿Cuáles son estos?

11. ¿Cuánto personal labora en este departamento?

12. ¿Cuál es la normatividad que sigue el departamento para la asignación de los recursos del Tribunal?

13. ¿Cuál es el órgano dedicado a la asignación de este recurso?

14. ¿Conoce la Ley General de Contabilidad Gubernamental?

15. ¿Aplica la LGCG actualmente? ¿Cómo?

16. ¿Cuánto personal labora en el STJET?

17. ¿En estos últimos años solicitó personal adicional? ¿Para qué áreas fue esto?

18. ¿Cuál es el procedimiento para la obtención del presupuesto del Tribunal?

19. ¿Los materiales que le asignan a este departamento son suficientes? ¿Por qué?

20. ¿Cuáles son los que requiere y en qué cantidades?

21. ¿Algún comentario o sugerencia que quisiera dar?

\section{Área de Recursos Humanos}

1. ¿Cuál es el número de personal en el STJET?

2. ¿Cuántos de estos son de base y cuántos temporales?

3. ¿Los empleados temporales para qué departamentos se contrataron?

4. ¿Cuál fue el presupuesto asignado al departamento?

5. ¿Cuál es el porcentaje del presupuesto establecido para nómina?

6. ¿Cuánto personal labora en este departamento?

7. ¿Los materiales que le asignan a este departamento son suficientes? ¿Por qué?

8. ¿Cuáles son los que requiere y en qué cantidades?

9. ¿Cuál es la normatividad que rige o sigue el departamento?

10. ¿Cuál es el procedimiento que sigue en el reclutamiento de planta laboral?

11. ¿Qué indicadores maneja en su departamento?

12. ¿Algún comentario o sugerencia que quisiera dar?

\section{Área de Almacén}

1. ¿Cuál es el presupuesto asignado al departamento?

2. ¿Cuántas personas laboran en el departamento?

3. ¿Cuál es la normatividad que rige o sigue el departamento?

4. ¿Los materiales que le asignan a este departamento son suficientes? ¿Por qué?

5. ¿Cuáles son los que requiere y en qué cantidades? 
6. ¿Cuál es el procedimiento que sigue el departamento para surtir de materiales a otros departamentos?

7. ¿Por qué nivel de gobierno o de qué forma recibe del presupuesto para el área?

8. ¿Cómo almacena los materiales que recibe en el área?

9. ¿Tiene métodos de control de inventarios? ¿Cuáles son?

10. ¿Maneja indicadores en su departamento?

11. ¿Hay algún procedimiento en caso de que el presupuesto asignado al departamento se haya agotado y se requiera comprar algún material?

12. ¿Las áreas tienen designado algún presupuesto en cuanto a la compra de materiales?

13. ¿A través de qué procedimiento se determina esto?

14. Algún comentario o sugerencia que quisiera dar

\section{Área de Nóminas}

1. ¿Usted conoce cuál es el presupuesto que se le ejerce al departamento de nóminas?

2. ¿Cuál es el departamento en el cual se ejerce la mayor parte de presupuesto?

3. ¿Es cierto que la mayor parte del presupuesto está asignado a Salas y Juzgados?

4. ¿Usted tiene en el departamento algún tipo de indicador que se maneje en el área?

5. ¿Qué normatividad sigue, por ejemplo, para el pago de la nómina?

6. ¿Todo el procedimiento de pago lo depositan vía bancaria?

7. ¿Usted maneja un sistema de información?

8. ¿En el sistema de nómina, ahí usted administra la alta o baja a trabajadores?

9. ¿A los sistemas que más usa como qué les faltaría o qué les agregaría usted?

10. ¿En cuánto a personal, usted piensa que sí necesita más personal?

11. ¿En cuanto a los materiales y suministros, los que les surten a ustedes sí son absolutamente suficientes?

12. ¿En cuanto a capacitación, con qué frecuencia se les brinda?

13. ¿Algún comentario además de lo referido a personal y a la capacitación?

Anexo 2. Matriz de evidencia entrevista

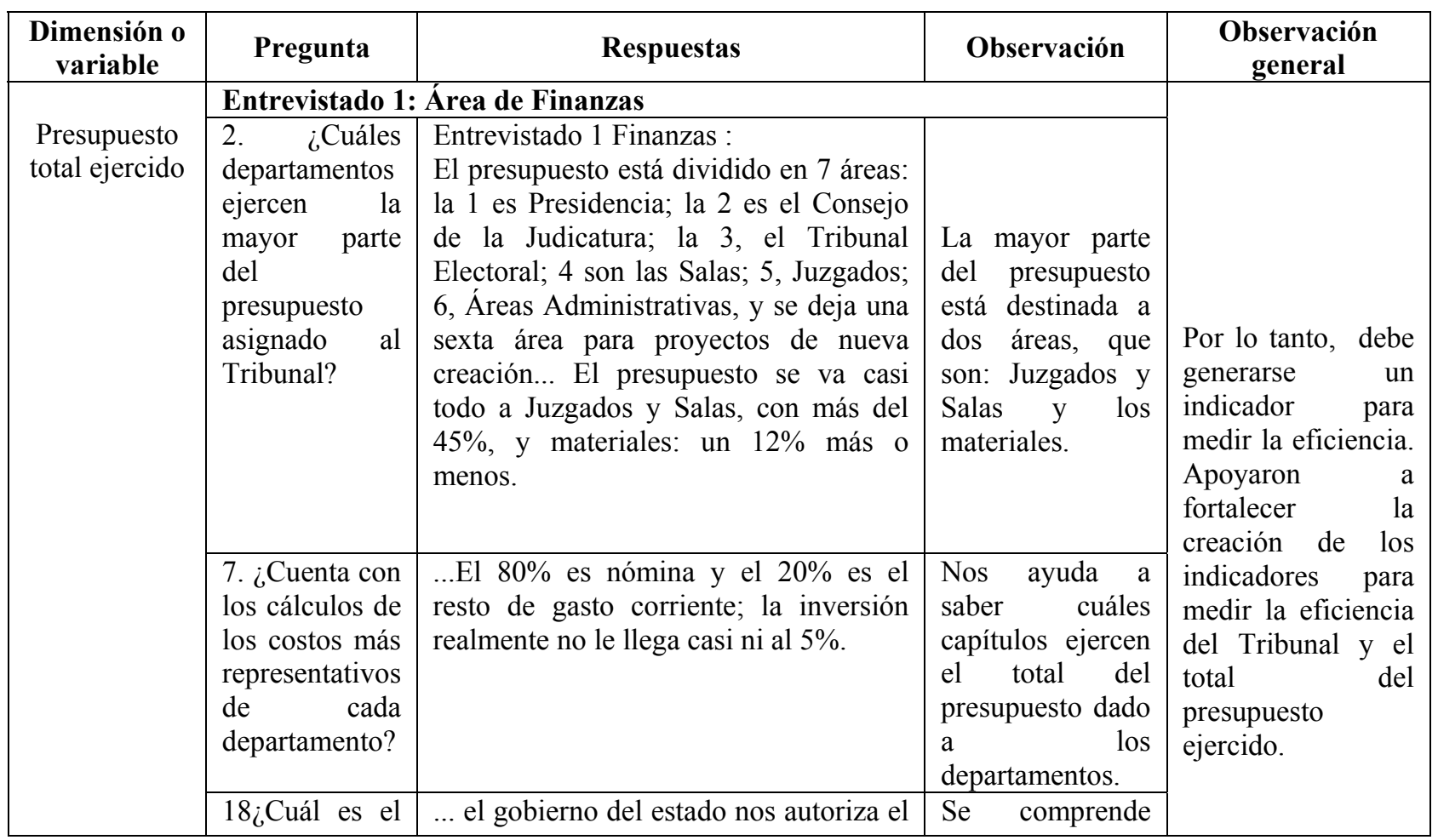




\begin{tabular}{|c|c|c|c|c|}
\hline & $\begin{array}{l}\text { procedimiento } \\
\text { para la } \\
\text { obtención del } \\
\text { presupuesto } \\
\text { del Tribunal? }\end{array}$ & $\begin{array}{l}\text { 1.3\% del presupuesto del Gobierno del } \\
\text { Estado... }\end{array}$ & $\begin{array}{l}\text { que el } \\
\text { presupuesto } \\
\text { ejercido total por } \\
\text { el STJE depende } \\
\text { del presupuesto } \\
\text { que se le autorice } \\
\text { al Estado. }\end{array}$ & \\
\hline & Entrevistado 2 & Área de Recursos Humanos & & \\
\hline & $\begin{array}{l}\text { 5. ¿Cuál es el } \\
\text { porcentaje del } \\
\text { presupuesto } \\
\text { establecido } \\
\text { para nómina? }\end{array}$ & $\begin{array}{l}\text { No se tiene, hay un área de nómina que } \\
\text { se encarga de proveer el presupuesto... }\end{array}$ & $\begin{array}{l}\text { No tienen } \\
\text { conocimiento del } \\
\text { presupuesto que } \\
\text { se gasta para el } \\
\text { área de nómina. }\end{array}$ & \\
\hline & Entrevistado 3 & Área de almacén & & \\
\hline & $\begin{array}{l}\text { 1. ¿Cuál es el } \\
\text { presupuesto } \\
\text { asignado al } \\
\text { departamento? }\end{array}$ & $\begin{array}{l}\text { Sí , y hay un tope o sea yo tengo un } \\
\text { tope de cierta cantidad, y si tengo cierta } \\
\text { necesidad de algún artículo, lo tengo } \\
\text { que sobrellevar; no me tengo que pasar } \\
\text { de la línea del tope de capital. }\end{array}$ & $\begin{array}{l}\text { Cuentan con el } \\
\text { conocimiento del } \\
\text { presupuesto. }\end{array}$ & \\
\hline & Entrevistado 4 & Área de Nóminas & & \\
\hline & $\begin{array}{l}\text { 1. ¿Usted } \\
\text { conoce cuál es } \\
\text { el presupuesto } \\
\text { que se le } \\
\text { ejerce al } \\
\text { Departamento } \\
\text { de Nóminas? }\end{array}$ & $\begin{array}{l}\text { De memoria no, pero en el sistema está } \\
\text { este. }\end{array}$ & $\begin{array}{l}\text { Tienen el } \\
\text { conocimiento del } \\
\text { presupuesto, pero } \\
\text { este tiene que ser } \\
\text { revisado en el } \\
\text { sistema del } \\
\text { organismo. }\end{array}$ & \\
\hline & $\begin{array}{l}\text { 2. ¿Cuál es el } \\
\text { departamento } \\
\text { en el cual se } \\
\text { ejerce la } \\
\text { mayor parte de } \\
\text { presupuesto? }\end{array}$ & $\begin{array}{l}\text {... que son Juzgados, Salas en todo el } \\
\text { estado. }\end{array}$ & $\begin{array}{l}\text { Se coincide con } \\
\text { otra área en que } \\
\text { el presupuesto } \\
\text { ejercido se va en } \\
\text { mayor parte a } \\
\text { Salas y Juzgados }\end{array}$ & \\
\hline 2. Eficiencia & Entrevistado 1 & Área de Finanzas & & \\
\hline $\begin{array}{l}\text { del } \\
\text { presupuesto } \\
\text { ejercido }\end{array}$ & $\begin{array}{l}\text { 9. ¿Maneja } \\
\text { indicadores de } \\
\text { gestión } \\
\text { financiera? }\end{array}$ & No, no los manejamos todavía. & $\begin{array}{l}\text { El no tener } \\
\text { indicadores } \\
\text { genera que no se } \\
\text { realice una } \\
\text { gestión adecuada } \\
\text { ya que no se } \\
\text { puede medir la } \\
\text { eficiencia de este } \\
\text { departamento. }\end{array}$ & $\begin{array}{l}\text { El presupuesto es } \\
\text { realizado de forma } \\
\text { tradicional, no se } \\
\text { atiende a objetivos } \\
\text { estratégicos o } \\
\text { metas }\end{array}$ \\
\hline & $\begin{array}{l}\text { 7. ¿Cuenta con } \\
\text { los cálculos de } \\
\text { los costos más } \\
\text { representativos } \\
\text { de cada } \\
\text { departamento? }\end{array}$ & $\begin{array}{l}\text { Los gastos más representativos son los } \\
\text { de nómina...; el segundo es servicios } \\
\text { generales, si yo siempre les digo: es un } \\
80-20 \text { casi } 80 \% \text {, o sea, } 78,79 \text { se va a } \\
\text { nómina; el } 12 \% \text { se va a servicios } \\
\text { generales; el } 5 \% \text { se va a materiales, y } \\
\text { suministros } 5-6 \% \text {. }\end{array}$ & $\begin{array}{l}\text { Solo en dos } \\
\text { departamentos se } \\
\text { destina casi la } \\
\text { totalidad del } \\
\text { presupuesto se } \\
\text { debe tener } \\
\text { indicadores de la } \\
\text { gestión que se } \\
\text { lleva con ellos. }\end{array}$ & $\begin{array}{l}\text { organizacionales, } \\
\text { es necesario medir } \\
\text { la eficiencia de } \\
\text { dicho presupuesto. }\end{array}$ \\
\hline & Entrevistado 2 & Área de Recursos Humanos & & \\
\hline & $\begin{array}{l}\text { 11. ¿Qué } \\
\text { indicadores }\end{array}$ & $\begin{array}{l}\text { No, digamos que aquí en el } \\
\text { departamento son funciones específicas }\end{array}$ & $\begin{array}{l}\text { El no tener } \\
\text { indicadores }\end{array}$ & \\
\hline
\end{tabular}




\begin{tabular}{|c|c|c|}
\hline $\begin{array}{l}\text { maneja en su } \\
\text { departamento? }\end{array}$ & $\begin{array}{l}\text { para cada una de las gentes }[\ldots] \text {, y los } \\
\text { indicadores son en sentido de dar } \\
\text { mayor eficiencia y eficacia de las } \\
\text { funciones que cada uno realiza, nuestro } \\
\text { modelo es que no tengamos rezagos en } \\
\text { la certificación de constancias o en los } \\
\text { trámites. }\end{array}$ & $\begin{array}{l}\text { genera que no se } \\
\text { realice una } \\
\text { gestión adecuada } \\
\text { ya que no se } \\
\text { puede medir la } \\
\text { eficiencia de este } \\
\text { departamento. }\end{array}$ \\
\hline \multicolumn{3}{|c|}{ Entrevistado 3: Área de Almacén } \\
\hline $\begin{array}{l}\text { 10. ¿Maneja } \\
\text { indicadores en } \\
\text { su } \\
\text { departamento? }\end{array}$ & $\begin{array}{l}\text { Tenemos un sistema [al] que nosotros } \\
\text { le pedimos, y nos da, }[\ldots] \text { ya tenemos } \\
\text { rato con ese sistema; y mucha } \\
\text { información que nosotros le pedimos, } \\
\text { nos la proporciona. }\end{array}$ & $\begin{array}{lr}\text { Cuentan } & \text { con } \\
\text { indicadores, pero } \\
\text { no establecidos; } \\
\text { solo } \\
\text { información que } \\
\text { requieren la } \\
\text { obtienen de lo } \\
\text { que arroja el } \\
\text { sistema. }\end{array}$ \\
\hline \multicolumn{3}{|c|}{ Entrevistado 4: Área de Nóminas } \\
\hline $\begin{array}{l}\text { 3. ¿Es cierto } \\
\text { que la mayor } \\
\text { parte del } \\
\text { presupuesto } \\
\text { está asignada a } \\
\text { Salas y } \\
\text { Juzgados? }\end{array}$ & $\begin{array}{l}\text { Sí, la mayoría de la plantilla se concreta } \\
\text { en lo que son Juzgados, Salas en todo el } \\
\text { estado. }\end{array}$ & $\begin{array}{l}\text { Las áreas que } \\
\text { más consumen el } \\
\text { presupuesto de } \\
\text { nuevo coinciden } \\
\text { en que [(..) son] } \\
\text { el Area de Salas } \\
\text { y Juzgados. }\end{array}$ \\
\hline $\begin{array}{l}\text { 4. ¿Usted tiene } \\
\text { en el } \\
\text { departamento } \\
\text { algún tipo de } \\
\text { indicador que } \\
\text { manejen en el } \\
\text { área? }\end{array}$ & $\begin{array}{l}\text { Sí, tenemos indicadores, pero no } \\
\text { institucionales; [son] más que nada de } \\
\text { control interno, que no la experiencia o } \\
\text { la rutina nos ha dado una pauta para } \\
\text { efectos de poder establecer un indicador } \\
\text { sin ser oficial sin ser institucional: } \\
\text { únicamente para el control interno. }\end{array}$ & $\begin{array}{l}\text { Tienen } \\
\text { indicadores, pero } \\
\text { no establecidos; } \\
\text { el no tener } \\
\text { indicadores } \\
\text { estudiados y } \\
\text { establecidos } \\
\text { puede generar a } \\
\text { que no haya una } \\
\text { eficiente gestión } \\
\text { del área. }\end{array}$ \\
\hline
\end{tabular}

Fuente: elaboración propia a partir de las entrevistas. 